\title{
The relationship between ethical climate of hospital and moral courage of nursing staff
}

\author{
Zeinab Taraz ${ }^{1}$, Laleh Loghmani ${ }^{2}$, Abbas Abbaszadeh ${ }^{3}$, Farzaneh Ahmadi ${ }^{4}$, Zahra Safavibiat $^{5}$, Fariba Borhani ${ }^{6}$
}

\begin{abstract}
Introduction: Courage is the ethical virtues that has special place in the nursing profession, whose role in patient support is highlighted. Courage virtues seems to grow with environmental reinforcement and social support. Hospital as a dynamic social institution has different forms of ethical climates. The purpose of this study was to determine the correlation between the ethical climate of the hospital and the moral courage of the nurses in one of the medical universities of Iran medical science's selected hospitals.

Method: This study is a descriptive-correlational study. The sample included 156 nurses who were selected by available method. A translated questionnaire of Olson's ethical climate and Sekerka's et al moral courage was used to collect data, in addition to demographic questions. The data were analyzed by SPSS software version 21 using descriptive and inferential statistics.

Findings: Nurses' perception of the ethical climates of the hospital was average (mean $=3.79, \mathrm{SD}=0.56$ ) and their courage was moderate (mean 3.87, $\mathrm{SD}=0.86)$. There was a significant positive correlation between nurses' ethical climates and their moral courage $(r=0.90, p<0.001)$.

Conclusion: According to the findings of this study, the moral courage virtue of nurses in ethical climates of support and care grows well. Therefore, to improve the role of protecting patients' rights and promote moral courage, attention should be paid to improving the ethical climates of the hospital. In addition, planners and nursing managers should consider arrangements for developing the ethical climates of the workplace.
\end{abstract}

Keywords: ethical climates, moral courage, nursing ethics, protection of patient rights

\section{INTRODUCTION}

One of the important responsibilities to protect the rights of patients is the duty of Advocacy. The preference of the patient's interests for individual interests and even their preferences to organizational interests requires the ethical virtue of courage. Moral courage is defined as continuous truth, defense of rights and commitment to moral principles in defending patients' rights, even in potential danger to their job position (Gallager, 2011). A nurse who has a moral courage, prefer commitment to patients in any case to their own interests (2). Ethical virtue of courage is a stimulant, which support person in the tiredness condition of helping others, and worries about the consequences of correct moral performance, and makes the nurse work to reach the result. The experience of many nurses includes the apparent disadvantages due to correct moral performance (3). These nurses may lose their motivation for moral work or decreases their willingness to serve patients, without ethical courage.

The moral organization should seek ways to strengthen the ethical virtue of courage. In an environment where moral courage is reduced, the rights of patients will be violated and the benefits of the patient will be trampled. The ways of promoting dare and adherence to ethics can lead to the promotion of ethical virtue of courage. Moral courage is

1 Student Research Committee; School of Nursing and Midwifery, Shahid Beheshti University of Medical Sciences; Tehran, Iran.

2 Assistant Professor, School of Nursing and Midwifery, Bam University of Medical Sciences, Bam, Iran.

3 Professor, Medical Ethics and Law Research Center, School of Nursing and Midwifery, Shahid Beheshti University of Medical Sciences; Tehran, Iran.

4 PhD student, Department of Biostatistics School of Paramedical Medicine, Shahid Beheshti University of Medical Sciences, Tehran, Iran.

5 Assistant professor, School of Nursing and Midwifery; Shahid Beheshti University of Medical Sciences; Tehran, Iran.

6 Associate professor, School of Nursing and Midwifery; Medical Ethics and Law Research Center, Shahid Beheshti University of Medical Sciences; Tehran, Iran.
Correspondence: Fariba Borhani

Associate professor, School of Nursing and Midwifery; Medical Ethics and Law Research Center, Shahid Beheshti University of Medical Sciences; Tehran, Iran.

E-mail:faribaborhani@msn.com

Received: 1 Mar 2018, Accepted: 17 Apr 2018

(C) 2019 by the authors; licensee Modestum Ltd., UK. This article is an open access article distributed under the terms and conditions of the Creative Commons Attribution License (http://creativecommons.org/licenses/by/4.0/). 
essential, and organizations in practice must provide the conditions for their employees to enjoy the ethical virtue of courage. Knowing the importance of moral courage and factors that enhance moral courage can help health care activists to work well in dealing with ethical challenges whether at the bedside of patients or in research and education activities (4).

The ethical climate of the organization seems to be effective on the employee's ethical courage. To mitigate moral problems, the organization can use the development of employee ethical courage. Although personal and professional factors influence the ethical courage of nurses, but in organizational terms, it seems that the organization's ethical climate can affect the ethical courage of employees. If such a hypothesis is correct, then it is the responsibility of hospital managers to develop ethical climate to promote moral courage so that work environments for nurses are in a state of professional commitment and ethical courage (5).

With all the importance of the ethical courage and ethical climate of the hospital, there have not yet been done enough studies in each of these cases, especially in relation between these two concepts. Major studies in this basis have addressed the ethical climate and ethical courage separately. However, if the research evidence considered hospital's ethical climate as of one of strengthening moral courage components, then it would be possible to turn strengthen nurses' ethical courage into an evidence-based subjects. This study has been done to represent the ethical climate in hospitals, which belong to one of the major universities in the capital of Iran, Shahid Beheshti University of Medical Sciences. Moreover, show nurses moral courage rate and the correlation between these two variables, considering the importance of ethical climate and the impact that can have on nurses' ethical courage.

\section{METHOD}

This descriptive-correlational study was conducted in 2016 in selected hospitals affiliated to Shahid Beheshti University of Medical Sciences, one of the major universities in the capital of Iran. Among the existing hospitals, three hospitals, Ayatollah Taleghani, Shahid Modarres and Loghman Hakim were randomly selected. All these three hospitals were General Hospitals and Medical Education Center. The required sample number was determined based on a pilot study of 150 people. Accordingly, 156 nurses were selected in the hospitals who were eligible for the study through sampling and from admissions departments except emergency departments and ICU, according to the quotas allocated to each hospital, department and different shifts. Attendance criteria were having at least a bachelor's degree in nursing, at least one year of experience in nursing and full-time nursing professions. The data-collecting tool was a three-part questionnaire. The first part contains demographic information that examines the characteristics of age, sex, type of service area, service life, and type of employment. The second part consists of a questionnaire "Ethical climate of the hospital." Olson in 1995 designed and validated this questionnaire to measure the ethical climate. Moreover, published in 1998 in the magazine Image and then in 2002 in the book Clinical Ethics Measuring Instruments. This questionnaire contains 26 questions in five areas of communication with colleagues, doctors, patients, hospital and managers. In addition, the range of scores for each question is based on Likert scale between one (almost never) and five (usually). Thus, the minimum total score for each person is 26 and the maximum is 130, and a higher score represents a more positive ethical climate of the environment. This questionnaire was translated into Farsi by Mobasher and his colleagues in 2004 and the optimum reliability (Cronbach's alpha coefficient $=0.92$ ) has been calculated. The third part included a moral courage questionnaire, which was designed by Sekerka et al. in 2009. This questionnaire contains 15 questions in five areas of ethics, multiple values, threat tolerance, moral sensitivity, and ethical goals. Each question based on Likert scale is scored from one (never) to five (always).

Validity of the questionnaires was confirmed by content validity method based on the opinion of ten professors in the field of ethics. To determine the reliability of the tools in this research, the questionnaires were distributed to 30 participating nurses and Cronbach's alpha coefficient was calculated to determine the internal consistency after completing the questionnaire. In this study, the internal correlation of "ethical climate of the Hospital" questionnaire using Cronbach's alpha for the whole tool was 0.92 , for "co-workers" factor 0.76 , for the "patients" factor 0.78 , for the "managers" factor 0.88 , for the "hospital" factor 0.80, and for "Doctors" factor was 0.75 . In addition, the internal correlation of the "moral courage" questionnaire using Cronbach's alpha for the whole tool was 0.8, for "ethical factor", 0.80 , for "multiple values" factor 0.7 , for "threat tolerance" factor 0.7 , for "moral sensitivity" factor 0.78 and for the "ethical goals" factor calculated 0.75 .

The questionnaire was given to the participants after obtaining the necessary permissions. Before distributing the questionnaires, received verbal testimonial from participants and pointed out that the information would be confidential and unnamed, and that all participants had complete freedom to enter and leave the study. In addition, during the 
Table 1: Mean and standard deviation of ethical climate and moral courage and its factors in terms of nurses' viewpoints

\begin{tabular}{|c|c|c|c|c|c|}
\hline Variable & No. & Mean & SD & Min. & Max. \\
\hline Ethical climate & 156 & 3.79 & 0.56 & 2.52 & 4.95 \\
\hline Communication with colleagues & 156 & 3.87 & 0.57 & 2.52 & 5.00 \\
\hline Communication with doctors & 156 & 3.75 & 0.51 & 2.67 & 3.75 \\
\hline Communication with patients & 156 & 3.79 & 0.68 & 2.42 & 5.00 \\
\hline Communication with the hospital & 156 & 3.77 & 0.57 & 2.46 & 4.17 \\
\hline Communication with managers & 156 & 3.79 & 0.72 & 1.71 & 4.17 \\
\hline Moral courage & 156 & 3.87 & 0.68 & 2.42 & 5.00 \\
\hline Moral factor & 156 & 3.91 & 0.47 & 2.67 & 4.96 \\
\hline Multiple Value & 156 & 3.51 & 0.59 & 2.42 & 5.00 \\
\hline Threat tolerance & 156 & 3.61 & 0.56 & 2.40 & 4.17 \\
\hline Moral sensitivity & 156 & 3.66 & 0.63 & 1.90 & 5.00 \\
\hline Achieving the goals & 156 & 3.53 & 0.74 & 1.46 & 5.00 \\
\hline
\end{tabular}

Table 2: Correlation between hospital ethical climate and moral courage of nurses

\begin{tabular}{lcc}
\hline Variable & Pearson Correlation & P-Value \\
\hline Ethical climate of hospital & 0.90 & $<0 / 001$ \\
\hline Moral courage of nurses & & $<$
\end{tabular}

completion of the questionnaire, the required explanations for each of the questions were given to the units under study. 185 questionnaires were distributed, of which 172 were returned, 91\% responded. Nine questionnaires was omitted due to the incomplete response to the terms. Finally, 156 questionnaires were used in the final examination.

Descriptive statistics were used to evaluate the frequency, mean and standard deviation of the studied variables and inferential statistics to investigate the correlation between ethical climate and ethical courage. In the following, using Kolmogorov-Smirnov test, were evaluated normality of variables. Regarding the normal distribution of variables, parametric tests such as Pearson correlation and independent t-test were performed using SPSS version 21 software.

\section{RESULTS}

In this study, participated 156 nurses with an average age of 30.97 and a standard deviation of 5.11 years, with an average work record of 7.09 and a standard deviation of 4.71 . Of the total samples, $67.9 \%$ were female and $53.2 \%$ were single. 45.5 percent were officially employed, 66 percent did not have a moral record, and 57.1 percent worked as turning shift. 68.6 percent were interested in nursing profession.

The average score for ethical climate of the hospital was 3.79 out of five with a standard deviation of 0.56 , which is assessed at an average level. In this study, the "Communication with colleagues" factor was found to be 3.87 and SD of 0.57 at the highest level and the "Communication with doctors" with mean of 3.75 and SD of 0.51 was at the lowest level (Table 1). It should be noted that the managers in this study means direct responsible for the nurse and not the administrative authorities or the head of the hospital.

In the present study, the average total moral courage among 156 nurses was 3.87 out of five with a standard deviation of 0.68 , which was assessed at a desirable level. "Moral factor" with an average of 3.91 and a standard deviation of 0.47 was the highest level of moral courage. "Multiple values" with a mean of 3.51 and a standard deviation of 0.59 was the lowest level of moral courage (Table 1).

Using Pearson Correlation test, there is a significant positive correlation $(p<0.001, r=0.92)$ between the ethical climate of the hospital and the moral courage of the nurses (Table 2).

\section{DISCUSSION AND CONCLUSION}

The results of this study showed that ethical climate with average score of 3.48 with a standard deviation of 0.61 was moderate, in Corley et al. (2005), this average was 3.33 out of 5 (6), in Ulrich et al. Equal was 2.6 (7) that is less than the average of this study. Perhaps the reason for this difference in working conditions and organizational culture in Iran hospitals with other countries. According to Shafipour et al. (2016) findings, in Iran, the average score of ethical climate was 3.5 (8) which is largely consistent with the results of the present study. Accordingly, the above average of ethical climate in Iran can be promising and similar results in Iranian studies indicate that the factors affecting the ethical climate of nurses working environment in Iran are similar. 
The results of the study on the dimensions of the ethical climate showed that the communication with colleagues had the highest average, which confirmed the positive atmosphere of cooperation between nurses. This finding could point to the coordination and collaboration within the group. There is a need for nurse co-operation to provide high quality care, which will increase the synergy of the forces and prevent potential harm to patients (9). In Hart's research (2005), the highest score in the ethical climate belongs to colleagues' factor and then managers, which is consistent with the results of the present study (10). The findings of this study showed that the cooperation between nurses and doctors (physician agents) is at the lowest level from the viewpoint of the examined nurses. Considering the importance of professional communication in providing high quality care, this issue should be further developed. Considering the fact that the services provided in the health system require interdisciplinary collaboration, this finding is alarming and it seems that nursing and medical services are provided in separate packages. The results of Shafipour et al. (2016) and Jolaee et al. (2013) in Iran suggest the appropriateness of the relationship between doctors and nurses (8 and 11). In such situation, not only the quality of care affected, the probability of complications such as the moral distress increases in doctors and nurses (12). In the study of Asterk (2003), the highest average is belonging to the "manager's factor" and the lowest is the "doctor's factor" (13). Which reveals a relatively similar pattern of organizational relationships between nurses and other colleagues in different cultures. It should be noted that the managers in this study means direct responsible for the nurse and not the administrative authorities or the head of the hospital, therefore, these results confirm the positive environment of cooperation between nurses. The findings of this study showed that the cooperation between the nurse and the doctors (physician agent) was at the lowest level from the viewpoint of the examined nurses.

The average score of moral courage of nurses in this study was 3.87 out of five with a standard deviation of 0.68 . This means that nurses consider their moral courage at a relatively high level. This finding is in line with the study of Mousavi et al in 2017. (14) The study of Day (2006) the moral courage of nurses reported poor (15). The reason for these differences seems to be factors such as ethical climate, organizational culture, managerial support, organizational support, fear of social isolation, organization's lack of acceptance, and group thinking on moral courage, which requires an examination of the relationship between such variables with moral courage.

In the present study, the "ethical factor" dimension in the questionnaire with a mean of 3.91 and SD of 0.47 was the highest among the factors. The ethical factor, which is the first dimension of moral courage, reflects a person's willingness to work for the right conduct and moral behavior in response to interactions with moral courage. This suggests that research units are a good moral agent to show moral courage. The high score of this factor indicates that nurses felt courage in their own virtues. The essence of nursing is care and requires nurses who are adorned with ethical virtues alongside with technical aspects for quality care (16). In addition, it seems that in educating nurses it is necessary to strengthen the morale of nurses (17). The results of a study in Iran showed that as nurses consider themselves as an ethical factor, they are more sensitive to providing care of patients (18). The increased nurses' moral sensitivity increases the quality of care and communication between nurses and patients (19).

The results of this study showed that the dimension of "multiple values" with a mean of 3.51 and SD of 0.04 is the lowest factor. This dimension identifies the individual's ability to choose a set of different values while making ethical decisions. Moreover, refers to the maintenance of beliefs in the heart despite external concerns and needs. This finding suggests that nurses do not have the ability to make ethical decisions and do not have the power to cope with organizational constraints and insistence on moral principles. Studies show that in the case of the inability of nurses in the right moral choices or the inability to emphasize ethical choices and ethics, they may be exposed to moral distress, and the quality of nursing care may be affected over time. In addition, in the long term, nurses tend to leave their careers (20).

The present study shows a significant correlation between ethical climate of the hospital and moral courage of nurses. $(p<0 / 001, r=0 / 92)$

Ethical virtues such as moral courage grow in the ethical climate, and whatever the virtuous people increase in the organization, their actions can be useful and effective. In such a situation, the likelihood of conflicts and moral challenges are reduced (1 and 21). Therefore, it is imperative to take decisions to increase the ability of nurses' ethical decision making, in order to reduce moral conflicts and help create a moral environment. The way will be paved for personal development and professionalism, and everyone benefits from physicians, nurses, staff and, ultimately, patients, in a place where, cooperation, empathy and ethical virtues grow every day. It seems that the role of ethical leaders can be very important in increasing the nurses' ability to make ethical decisions and create an ethical climate (22). Finally, when nurses can gain courage virtue, they can play their supportive role for patients better, and in such situation, patients feel safe and can trust the nurses (23). 


\section{CONCLUSION}

Nowadays, health service providers have found a lot of complexity, various advanced equipment and the many people present in these organizations have an impact on the ethical climate of organizations. Ethical climate is considered as the personality of the organization. The results of this study also showed that the higher ethical climate score of the hospital lead to the more ethical virtue of courage in nurses. This will ultimately lead patients to better care and reduce the risk of physical, mental and mental harm to both patients and the health care provider. Therefore, nursing managers can use the operational features to improve ethical climate and ethical courage of nurses to get synergy between these two variables in order to achieve satisfaction, trust and confidence of patients in the nursing profession and health services organizations.

\section{REFERENCES}

1. Gallager A. Moral distress and moral courage in everyday Nursing practice. Online J Issues Nurse. 2010;16(2):8.

2. Sekerka EL, Bagozzi RP, Charnigo R. Facing ethical challenges in the workplace: conceptualizing and measuring professional moral courage. J Bus Ethics. 2009;89:79-565. https://doi.org/10.1007/s10551-008-0017-5

3. LaSala CA, Bjarnason D. Creating Workplace Environments that Support Moral Courage. OJIN: The Online Journal of Issues in Nursing. 2010;15(3).

4. Lachman VD, Murray JS, Iseminger K, Ganske KM. Doing the right thing: pathways to moral courage. Am Nurs Today. 2012;11(11):87-1996.

5. Suhonen $R$, Stolt $M$, Virtanen $H$, Leino-Kilipo $H$. Organizational ethics: a literature review. Nurse Ethics. 2011;18(3):283-303. https://doi.org/10.1177/0969733011401123

6. Corley MC, Minicik P, Elswick RK, Jacobs M. Nurse moral distress and ethical work environment. Nursing Ethics. 2005;12(4):381-390. https://doi.org/10.1191/0969733005ne809oa

7. Ulrich et al. Ethical climate, ethics stress and the job satisfaction of nurses and social workers in the United States. Social Science and Medicine. 2007;65(8):1708-1719. https://doi.org/10.1016/j.socscimed.2007.05.050

8. Shafipour V, Yaghobian M, Shafipour L, Heidari MR. Nurses' perception of the ethical climate in the Iranian hospital environment. Journal of Nursing and Midwifery Sciences. 2016;3(4):37-43. https://doi.org/10.18869/acadpub.jnms.3.4.37

9. Farokhzadian, J., Nayeri, N.D., Borhani, F. Rocky milieu: challenges of effective integration of clinical risk management into hospitals in Iran Int J Qual Stud Health Well-being. 2015 May 11;10:27040.

10. Hart SE. Hospital ethical climate $s$ and registered nurses `turnover intentions. Journal of Nursing Scholarship. 2005;37(2):173-177. https://doi.org/10.1111/j.1547-5069.2005.00030.x

11.

12. Joolaee $S$, Jalili HR, Rafii $F$, Hajibabaee $F$, Haghani $H$. The relationship between ethical climate at work and job satisfaction among nurses in Tehran. Indian J Med Ethics. 2013;10(4):238-42. https://doi.org/10.20529/IJME.2013.072

13. Abbasi, M., Nejadsarvari, N., Kiani, M., (...), Tavaokkoli, S.N., Rasouli, H. Moral distress in physicians practicing in hospitals affiliated to medical sciences universities Iran Red Crescent Med J. 2014 Oct 5;16(10):e18797. https://doi.org/10.5812/ircmj.18797

14. Moosavi, S.S., Borhani, F., Abbaszadeh, A. The moral courage of nurses employed in hospitals affiliated to Shahid Beheshti University of Medical Sciences. Hayat, Journal of School of Nursing and Midwifery, Tehran University of Medical Sciences. 2016;22(4):339-349. (Persian)

15. Day L. Courage as a virtue necessary to good nursing practice. American Journal of Critical Care. 2007;16(6):613 $-616$.

16. Shahriari M, Mohammadi E, Fooladi MM, Abbaszadeh A, Bahrami M. Proposing Codes of Ethics for Iranian Nurses: A Mixed Methods Study. Journal of Mixed Methods Research. 2015;10(4):352-366. https://doi.org/10.1177/1558689815570375

17. Bahrieni F, Azodi P, Hajivandi A, Jahanpour F. The effect of education in nurse's moral sensitivity. Pharm. Sci. \& Res. 2017;9(10):1817-21.

18. Borhani F, Keshtgar M, Abbaszadeh A. Moral self-concept and moral sensitivity in Iranian nurses. J Med Ethics Hist Med. 2015 Apr 4;8:4. 
19. Zamanzadeh V, Rassouli M, Abbaszadeh A, Nikanfar A, Alavi-Majd H, Ghahramanian A. Factors Influencing Communication Between the Patients with Cancer and their Nurses in Oncology Wards. Indian Journal of Palliative Care. 2014;20(1):12-20. https://doi.org/10.4103/0973-1075.125549

20. Borhani F, Abbaszadeh A, Nakhaee N, Roshanzadeh M. The relationship between moral distress, professional stress, and intent to stay in the nursing profession. Journal of Medical Ethics and History of Medicine. 2014;7:3.

21. Sekerka EL, Bagozzi RP and Charnigo R. Facing ethical challenges in the workplace: conceptualizing and measuring professional moral courage. J Bus Ethics. 2009;89:79-565.

22. Esmaelzadeh F, Abbaszadeh A, Borhani F, Peyrovi H. Strengthening ethical decision-making: the experience of Iranian nurses. Nurs Manag (Harrow). 2017 Sep 28;24(6):33-39. https://doi.org/10.7748/nm.2017.e1610

23. Davoodvand S, Abbaszadeh A, Ahmadi F. Patient advocacy from the clinical nurses' viewpoint: a qualitative study. J Med Ethics Hist Med. 2016;11(9):5.

$\diamond \diamond \diamond \diamond \diamond \diamond \diamond$

http://www.ejgm.co.uk 